Springer Proceedings in Mathematics \& Statistics

Sandra Pinelas

Zuzana Došlá

Ondřej Došlý

Peter E. Kloeden Editors

Differential

and Difference

Equations with

Applications

ICDDEA, Amadora, Portugal, May 2015,

Selected Contributions

Springer 


\section{Springer Proceedings in Mathematics \& Statistics}

Volume 164

More information about this series at http://www.springer.com/series/10533 


\section{Springer Proceedings in Mathematics \& Statistics}

This book series features volumes composed of select contributions from workshops and conferences in all areas of current research in mathematics and statistics, including OR and optimization. In addition to an overall evaluation of the interest, scientific quality, and timeliness of each proposal at the hands of the publisher, individual contributions are all refereed to the high quality standards of leading journals in the field. Thus, this series provides the research community with well-edited, authoritative reports on developments in the most exciting areas of mathematical and statistical research today. 
Sandra Pinelas • Zuzana Došlá

Ondřej Došlý • Peter E. Kloeden

Editors

\section{Differential and Difference Equations with Applications}

ICDDEA, Amadora, Portugal, May 2015, Selected Contributions

型 Springer 


\section{Editors}

Sandra Pinelas

Departamento de Ciências Exatas e Naturais

Academia Militar

Amadora, Portugal

Ondřej Došlý

Department of Mathematics and Statistics

Masaryk University

Brno, Czech Republic

\section{Zuzana Došlá}

Department of Mathematics and Statistics

Masaryk University

Brno, Czech Republic

Peter E. Kloeden

School of Mathematics and Stastistics

Huazhong University of Science

and Technology

Wuhan China

ISSN 2194-1009

ISSN 2194-1017 (electronic)

Springer Proceedings in Mathematics \& Statistics

ISBN 978-3-319-32855-3

DOI 10.1007/978-3-319-32857-7

Library of Congress Control Number: 2016947464

(c) Springer International Publishing Switzerland 2016

This work is subject to copyright. All rights are reserved by the Publisher, whether the whole or part of the material is concerned, specifically the rights of translation, reprinting, reuse of illustrations, recitation, broadcasting, reproduction on microfilms or in any other physical way, and transmission or information storage and retrieval, electronic adaptation, computer software, or by similar or dissimilar methodology now known or hereafter developed.

The use of general descriptive names, registered names, trademarks, service marks, etc. in this publication does not imply, even in the absence of a specific statement, that such names are exempt from the relevant protective laws and regulations and therefore free for general use.

The publisher, the authors and the editors are safe to assume that the advice and information in this book are believed to be true and accurate at the date of publication. Neither the publisher nor the authors or the editors give a warranty, express or implied, with respect to the material contained herein or for any errors or omissions that may have been made.

Printed on acid-free paper

This Springer imprint is published by Springer Nature

The registered company is Springer International Publishing AG Switzerland 


\title{
On a One-Equation Turbulent Model with Feedbacks
}

\author{
H.B. de Oliveira and A. Paiva
}

\begin{abstract}
A one-equation turbulent model is derived in this work on the basis of the approach used for the $k$-epsilon model. The novelty of the model consists in the consideration of a general feedback forces field in the momentum equation and a rather general turbulent dissipation function in the equation for the turbulent kinetic energy. For the steady-state associated boundary value problem, we prove the uniqueness of weak solutions under monotonous conditions on the feedbacks and smallness conditions on the solutions to the problem. We also discuss the existence of weak solutions and issues related with the higher integrability of the solutions gradients.
\end{abstract}

Keywords Turbulence $\bullet k$-epsilon model $\bullet$ Feedback forces $\bullet$ Uniqueness

Mathematics Subject Classification (2010): 76F60, 93A30, 35Q35, 76D03

\section{Introduction}

The Navier-Stokes equations were proposed by Navier in 1822, and later on, in 1845 , due to the clarifying work made by Stokes, these equations found a phenomenological justification on the basis of the principles of fluid mechanics. Since then, these equations are used to describe Newtonian fluid flows, which, in the case of incompressible and homogeneous fluids, can be written as

\footnotetext{
H.B. de Oliveira

Faculty of Sciences and Technology, Universidade do Algarve, Faro, Portugal

Centro de Matemática, Aplicações Fundamentais e Investigação Operacional (CMAFCIO),

Universidade de Lisboa, Lisboa, Portugal

e-mail: holivei@ualg.pt
}

A. Paiva $(\square)$

Faculty of Sciences and Technology, Universidade do Algarve, Faro, Portugal

e-mail: ana-paiva@hotmail.com 


$$
\begin{aligned}
& \operatorname{div} \mathbf{u}=0, \\
& \frac{\partial \mathbf{u}}{\partial t}+\operatorname{div}(\mathbf{u} \otimes \mathbf{u})=\mathbf{f}-\frac{1}{\rho} \nabla p+v \mathbf{D}(\mathbf{u}), \quad \mathbf{D}(\mathbf{u})=\frac{1}{2}\left(\nabla \mathbf{u}+\nabla \mathbf{u}^{T}\right),
\end{aligned}
$$

where $\mathbf{u}=\left(u_{1}, u_{2}, u_{3}\right)$ is the velocity field, $p$ is the pressure, $\rho$ is the constant (positive) density, and $\mathbf{f}$ denotes the external forces field. The tensor $\mathbf{D}(\mathbf{u})$ is the symmetric part of $\nabla \mathbf{u}$ and accounts for the different strains in the fluid. The positive factor $v$ expresses the ratio of the internal forces in the fluid, called dynamic viscosity, to the mass density $\rho$, and is usually called kinematic viscosity. In 1883, Reynolds has succeeded to prove the importance of a threshold value separating the laminar flow regime from the turbulent one within a similar fluid. Nowadays, this value is known as the Reynolds number, and it is usually defined as the ratio of inertial forces to viscous forces $R e=\frac{u(l) l}{v} \equiv \frac{(\mathbf{u} \cdot \nabla) \mathbf{u}}{v \Delta \mathbf{u}}$, where $l$ and $u(l)$ are characteristic length and velocity scales. It was Stokes, even before Reynolds, who observed the inadequacy of (1) and (2) to model certain flow regimes that could probably result from eddies which rendered the motion more chaotic. However, it seems to have been Reynolds the first to study the mechanical significance of the existence of such eddies. The approach made by Reynolds was to assume that the flow has two different scales, leading to the supposition that it is possible to decompose the quantities in the Navier-Stokes equations in average and fluctuating, or aleatory, values (Reynolds hypothesis). The idea associated to this decomposition was to filter the Navier-Stokes equations in time intervals large enough, in comparison to the temporal scale of the flow, but small enough in comparison with the time scale of the average of the flow. Therefore, the velocity of a molecule was decomposed into two components:

$$
\mathbf{u}=\overline{\mathbf{u}}+\mathbf{u}^{\prime}
$$

where $\mathbf{u}^{\prime}$ represents the fluctuating, or relative velocity, and $\overline{\mathbf{u}}$ represents an average velocity. Underlying this decomposition is a filter, or an average, concept that can be mathematically defined as a Reynolds operator [15], i.e., an operator $\mathscr{R}: \mathbb{R}^{3} \longrightarrow \mathbb{R}^{3}$ defined by $\mathscr{R}(\mathbf{u})=\overline{\mathbf{u}}$ and satisfying to the following properties:

$$
\begin{aligned}
\mathscr{R}(\mathbf{u}+\lambda \mathbf{v}) & =\mathscr{R}(\mathbf{u})+\lambda \mathscr{R}(\mathbf{v}) \quad \forall \mathbf{u}, \mathbf{v} \in \mathbb{R}^{3} \quad \forall \lambda \in \mathbb{R} ; \\
\mathscr{R}(\mathscr{R}(\mathbf{u})) & =\mathscr{R}(\mathbf{u}) \quad \forall \mathbf{u} \in \mathbb{R}^{3} ; \\
\mathscr{R}(\partial \mathbf{u}) & =\partial(\mathscr{R}(\mathbf{u})) \quad \forall \mathbf{u} \in \mathbb{R}^{3} ; \\
\mathscr{R}(\mathbf{u} \otimes \mathbf{v}) & =\mathscr{R}(\mathbf{u}) \otimes \mathscr{R}(\mathbf{v})+\mathscr{R}((\mathbf{u}-\mathscr{R}(\mathbf{u})) \otimes(\mathbf{v}-\mathscr{R}(\mathbf{v}))) \quad \forall \mathbf{u}, \mathbf{v} \in \mathbb{R}^{3} .
\end{aligned}
$$

Observe that (5) implies that $\mathscr{R}\left(\mathbf{u}^{\prime}\right)=\mathbf{0}$ for any $\mathbf{u} \in \mathbb{R}^{3}$, and from (7) we have $\mathscr{R}(\mathbf{u} \otimes \mathscr{R}(\mathbf{v}))=\mathscr{R}(\mathbf{u}) \otimes \mathscr{R}(\mathbf{v})$ and $\mathscr{R}(\mathscr{R}(\mathbf{u}) \otimes \mathbf{v})=\mathscr{R}(\mathbf{u}) \otimes \mathscr{R}(\mathbf{v})$ which makes $\mathscr{R}(\mathbf{u} \otimes \mathscr{R}(\mathbf{v}))=\mathscr{R}(\mathscr{R}(\mathbf{u}) \otimes \mathbf{v})$. Since the tensorial product is not commutative, we have, in general, $\mathscr{R}(\mathbf{u} \otimes \mathscr{R}(\mathbf{v})) \neq \mathscr{R}(\mathbf{v} \otimes \mathscr{R}(\mathbf{u}))$. However, this inequality is 
not observed when the Reynolds averaged Navier-Stokes equations (RANS) are derived in the scalar form. The definition of Reynolds operator described above is used to filter the Navier-Stokes equations (1) and (2) in a domain $\Omega \subset \mathrm{R}^{3}$, which represents the volume occupied by the fluid on the time $t \in[0, T]$. After some algebraic manipulations, we obtain the so-called RANS equations

$$
\begin{aligned}
\operatorname{div} \overline{\mathbf{u}} & =0, \\
\frac{\partial \overline{\mathbf{u}}}{\partial t}+\operatorname{div}(\overline{\mathbf{u}} \otimes \overline{\mathbf{u}}) & =\overline{\mathbf{f}}-\frac{1}{\rho} \nabla \bar{p}+v \operatorname{div} \mathbf{D}(\overline{\mathbf{u}})-\operatorname{div}\left(\overline{\mathbf{u}^{\prime} \otimes \overline{\mathbf{u}}^{\prime}}\right) .
\end{aligned}
$$

Equation (9) looks the same as the momentum equation (2), with the addition of a term involving the average of a product of fluctuating parts of the velocity. The additional term

$$
\mathbf{R}:=-\overline{\mathbf{u}^{\prime} \otimes \mathbf{u}^{\prime}},
$$

often called the Reynolds stress tensor, can be seen as the average of changes in $\mathbf{u}^{\prime}$ due to the particle transport with the fluid movement. Therefore, the tensor (10) acts like an effective stress and cannot be determined from the classical principles. As we do not have any way to know directly its magnitude, the modeling of its effect is usually done in terms of known quantities or quantities that we can determine. This is known in the literature as the closing problem of turbulence, and, as a result, many schemes have been developed to approximate the Reynolds stress tensor.

\section{The $k-\epsilon$ Turbulent Model}

Reynolds has made experiments suggesting that the tensor (10) was somehow related with $\nabla \overline{\mathbf{u}}$, which, by reasons of symmetry, can now be considered in the form $\mathbf{R}=\mathbf{F}(\mathbf{D}(\overline{\mathbf{u}}))$. However, the application $\mathbf{F}$ cannot be arbitrarily chosen, because the model should give the same results regardless of the considered referential. In analogy with the Stokes law for laminar flows, Boussinesq has proposed that $\mathbf{R}=v_{T} \mathbf{D}(\overline{\mathbf{u}})$ (Boussinesq turbulence hypothesis), where $v_{T}$ was denoted by eddy or turbulent viscosity. By a simple comparison of the traces of the last expression with (10), it can be readily seen that the Boussinesq hypothesis must be rewritten in the form

$$
\mathbf{R}=-\frac{2}{3} k \mathbf{I}+v_{T} \mathbf{D}(\overline{\mathbf{u}}), \quad k:=\frac{1}{2} \overline{\left|\mathbf{u}^{\prime}\right|^{2}},
$$

where $k$ is called turbulent kinetic energy, a new unknown in the problem that needs also to be modeled. In 1942, Kolmogorov [10] proposed a model in which the turbulence was described by $v_{T}=\rho \frac{k}{f}$ and $l=k^{\frac{1}{2}} / f$, where $l$ is a length scale, suggesting that $k$ and $f$, the characteristic frequency of the energy-containing 
movements, should be determined by transport equations. Inspired by the previous model of his own, Prandtl [14] proposed, in 1945, that $v_{T}=\rho k^{\frac{1}{2}} l$, suggesting also that the turbulent kinetic energy was determined from a transport equation, but the length scale $l$ should be algebraically prescribed. Later on, during the 1970 decade, Launder and Spalding [11] observed the importance of the turbulent dissipation $\epsilon:=v \overline{\left|\nabla \mathbf{u}^{\prime}\right|^{2}}$, a new quantity, in determining the rate of dissipation of the turbulent kinetic energy in the turbulent flow process, which, again by means of symmetry, can be written as $\epsilon:=v \overline{\left|\mathbf{D}\left(\mathbf{u}^{\prime}\right)\right|^{2}}$. The turbulent dissipation $\epsilon$ is determined by the first process in the energy cascade, which consists in the transfer of energy from the largest eddies to the smaller ones. Assuming these large eddies are characterized by length scale $l_{0}$, velocity scale $u_{0}$, and time scale $t_{0}=l_{0} / u_{0}$ and have energy of $1 / 2 \rho u_{0}^{2}$, then the rate of transfer of energy can be supposed to scale as $u_{0}^{2} / t_{0}=u_{0}^{3} / l_{0}$. Consequently, $\epsilon$ scales as $u_{0}^{3} / l_{0}$ and independently of $v$. Therefore, it is reasonable to model $\epsilon$ and consequently the turbulent viscosity (in view of Prandtl's hypothesis), as

$$
\epsilon=C_{D} \frac{k^{\frac{3}{2}}}{l}, \quad v_{T}=\rho k^{\frac{1}{2}} l \Rightarrow v_{T}=C_{\mu} \frac{k^{2}}{\epsilon}, \quad \text { where } \epsilon:=v \overline{\left|\mathbf{D}\left(\mathbf{u}^{\prime}\right)\right|^{2}},
$$

$C_{D}$ is a closure constant, and $C_{\mu}$ is a constant related with the kinematic viscosity and determined by experimental measures of $k$ and $\epsilon$. To derive an equation for the transport of the turbulent kinetic energy, we start by considering (1) the velocity field decomposed in the form (3). Then, subtracting (9) to this equation, we obtain

$$
\operatorname{div} \mathbf{u}^{\prime}=0
$$

Likewise, we subtract the RANS equations (9) to the momentum equation (2), where all the quantities are decomposed as in (3). Then, we multiply the resulting equation by $\mathbf{u}^{\prime}$ and we apply the filter produced by the Reynolds operator. Using the properties set forth at (4)-(7) and some vectorial calculus together with (10), (11) 2 , and (13), we obtain

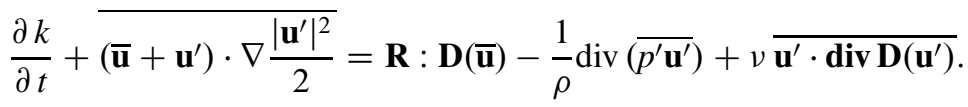

By using the hypothesis that convection by random fields produces diffusion for the mean [12], the second term of the left-hand side of (14) can be approximated by $\overline{\mathbf{u}} \cdot \nabla k-\operatorname{div}\left(v_{D} \nabla k\right)$, where $v_{D}:=v_{D}(k, \epsilon)$ is the turbulent diffusivity. For the last two terms, it is used an ergodicity hypothesis [4] asserting that, over a long period of time, the remaining time on a given region in space is proportional to the region volume. This allows us to use the approximations $\overline{\mathbf{u}^{\prime} \cdot \operatorname{div} \mathbf{D}\left(\mathbf{u}^{\prime}\right)} \simeq \overline{\left|\mathbf{D}\left(\mathbf{u}^{\prime}\right)\right|^{2}} \simeq \epsilon$ and $\operatorname{div}\left(\overline{p^{\prime} \mathbf{u}^{\prime}}\right) \simeq 0$. Using this information, we obtain the following transport equation for $k$ 


$$
\frac{\partial k}{\partial t}+\overline{\mathbf{u}} \cdot \nabla k=\operatorname{div}\left(v_{D} \nabla k\right)+v_{T}|\mathbf{D}(\overline{\mathbf{u}})|^{2}-\epsilon, \quad v_{D}:=v_{D}(k, \epsilon)
$$

The usual process to derive an equation for the evolution of the turbulent dissipation $\epsilon$ starts by applying the rotational to the RANS equation (9), and then working with calculus tools, the following is obtained:

$$
\begin{aligned}
\frac{\partial \varepsilon}{\partial t} & -2 v\left(\overline{\operatorname{rot} \mathbf{u}^{\prime} \cdot \operatorname{rot}\left(\mathbf{u}^{\prime} \times \operatorname{rot} \overline{\mathbf{u}}\right)}\right)-2 v\left(\overline{\left.\operatorname{\operatorname {rot}\mathbf {u}^{\prime }\otimes \operatorname {rot}\mathbf {u}^{\prime }}: \nabla \overline{\mathbf{u}}\right)}\right. \\
& -2 v\left(\overline{\left(\operatorname{rot} \mathbf{u}^{\prime} \otimes \operatorname{rot} \mathbf{u}^{\prime}\right)^{\prime}: \nabla \mathbf{u}^{\prime}}\right)+\left(\overline{\left(\overline{\mathbf{u}}+\mathbf{u}^{\prime}\right) \cdot v \nabla\left|\operatorname{rot} \mathbf{u}^{\prime}\right|^{2}}\right)=-2 v^{2} \overline{\left|\nabla \operatorname{rot} \mathbf{u}^{\prime}\right|^{2}} .
\end{aligned}
$$

The second term of the left-hand side can be neglected because the terms involved approximately cancel one each other. Using an arguing similar to the Boussinesq hypothesis, the second term is approximated by $2 \nu C k|\mathbf{D}(\overline{\mathbf{u}})|^{2}$. The last term is approximated by $\overline{\mathbf{u}} \cdot \nabla \varepsilon-\operatorname{div}\left(v_{D} \nabla \varepsilon\right)$ by the application of the convection-diffusion hypothesis [12] similarly as it was done for the $k$-equation. Finally, the fourth term on the left-hand side and the term on the right-hand side are usually approximated by $C \frac{\varepsilon^{2}}{k}$ to avoid the need of another equation in order to close the problem [4, 12]. After all, we arrive in the following evolution equation:

$$
\frac{\partial \varepsilon}{\partial t}+\overline{\mathbf{u}} \cdot \nabla \varepsilon=\operatorname{div}\left(v_{D}(k, \varepsilon) \nabla \varepsilon\right)+C_{1} k|\mathbf{D}(\overline{\mathbf{u}})|^{2}+C_{2} \frac{\varepsilon^{2}}{k},
$$

where $C_{1}$ and $C_{2}$ are positive constants that can be determined from the experiments.

\section{Feedback Forces Fields}

In this section we consider, for simplicity, 1-equation models comprised by Eqs. (8), (9), (11), (12), and (15), and we assume the turbulent dissipation $\epsilon$ depends only on $k$. Observe that the consideration of 1-equation models is acceptable in the sense that the equation for $\epsilon$ may be discarded by prescribing an appropriate length scale. This assumption has also implications on the turbulent viscosity and on the turbulent diffusivity, defined at (12) and (15), in the sense that now they only depend on $k$. We consider here the case when the external forces field depends on the own velocity, i.e., we assume that the vector field $\mathbf{f}$, in the momentum equation (2), is replaced by

$$
\mathbf{g}-\mathbf{f}(\mathbf{u})
$$

Now, in (18), $\mathbf{g}$ is an external forcing term that depends only on the space and time variables, and $\mathbf{f}(\mathbf{u})$ is the feedback forces field that may have different signs, according to each application where it is considered. Probably the best-known situation happens for fluid flows in a rotating frame, where the Coriolis force 
$\mathbf{f}(\mathbf{u})=2 \Omega \times \mathbf{u}$ must be considered, being $\Omega$, here, the angular velocity vector. Another example is the Lorentz force $\mathbf{f}(\mathbf{u}, \mathbf{B})=-\mathbf{J} \times \mathbf{B}$ considered to model turbulent flows controlled by a magnetic field $\mathbf{B}$, where $\mathbf{J}$ is the total electric current intensity, given by Ohm's law $\mathbf{J}=\sigma(-\nabla \Phi+\mathbf{u} \times \mathbf{B})$. Here, $\sigma$ is the conductivity, a material-dependent parameter, and $\Phi$ is the electric potential, which in turn satisfies to the Poisson equation $\triangle \Phi=\operatorname{div}(\mathbf{u} \times \mathbf{B})$ (see, e.g., [9]). However, our main motivation comes from the study of flows through porous media. In this field of the applications, it is important to consider the Darcy and Forchheimer terms to model the drag due to the flow through the porous medium.

Here, we gather this drag in the function $\mathbf{f}(\mathbf{u})=C_{D} \mathbf{u}+C_{F}|\mathbf{u}| \mathbf{u}$, where $C_{D}$ and $C_{F}$ are the Darcy and Forchheimer parameters, positive constants that depend on the permeability and porosity of the medium. The mathematical modeling of turbulence in porous media considers the simultaneous application of time and volume-average operators. When this procedure is applied to the continuity and momentum equations, they come as in (8) and (9), with the peculiarity that the Darcy and Forchheimer terms come in the form $\mathbf{f}(\overline{\mathbf{u}})=C_{D} \overline{\mathbf{u}}+C_{F}|\overline{\mathbf{u}}| \overline{\mathbf{u}}$. This procedure is being applied to many situations of turbulent fluids through porous media, as is the case of turbulent combustion in porous media or turbulent impinging jets in porous media (see, e.g., [5]). The underlying idea of considering this double-decomposition concept corresponds, in a certain sense, to consider a feedback forces field

$$
\mathbf{f}(\mathbf{u})=\overline{\mathbf{f}(\mathbf{u})}+\mathbf{f}(\mathbf{u})^{\prime}, \quad \overline{\mathbf{f}(\mathbf{u})}=\mathbf{f}(\overline{\mathbf{u}}), \quad \overline{\mathbf{f}(\mathbf{u})^{\prime} \cdot \mathbf{u}^{\prime}}=h(|\overline{\mathbf{u}}|) k
$$

The best example of this situation is a drag's force purely Darcy $\mathbf{f}(\mathbf{u})=C_{D} \mathbf{u}$ for which $h(|\overline{\mathbf{u}}|)=2 C_{D}$. A more complex example of a feedback forces field satisfying to (19) is given by the generalized Forchheimer force

$$
\mathbf{f}(\mathbf{u})=h(|\mathbf{u}|) \mathbf{u}, \quad h(|\mathbf{u}|)=|\overline{\mathbf{u}}|^{2 n}, \quad n \in \mathbb{N} .
$$

The Reynolds averaged process for the momentum equation, considered with a feedback forces field satisfying to (18) and (19) and assuming the Reynolds stresses, is given by $\mathbf{R}=v_{T} \overline{\mathbf{D}}$, and the turbulent viscosity, defined by (12), depends only on $k$, leads us to

$$
\frac{\partial \overline{\mathbf{u}}}{\partial t}+\operatorname{div}(\overline{\mathbf{u}} \otimes \overline{\mathbf{u}})=\overline{\mathbf{g}}-\mathbf{f}(\overline{\mathbf{u}})-\frac{1}{\rho} \nabla \bar{p}+\operatorname{div}\left(\left(v+v_{T}(k)\right) \mathbf{D}(\overline{\mathbf{u}})\right) .
$$

The same procedure used to derive the $k$-equation, and assuming, in addition, the turbulent diffusivity, given by (15), depends also only on $k$, allows us to write

$$
\frac{\partial k}{\partial t}+\overline{\mathbf{u}} \cdot \nabla k=\operatorname{div}\left(v_{D}(k) \nabla k\right)+v_{T}(k)|\mathbf{D}(\overline{\mathbf{u}})|^{2}+h(|\overline{\mathbf{u}}|) k-\epsilon(k) .
$$

Although the term $h(|\overline{\mathbf{u}}|) k$ does not bring any difficulty to our analysis, we may avoid its presence in Eq. (22) by considering a general feedback forces field satisfying to (19), but with (19) $)_{3}$ replaced by

$$
\overline{\mathbf{f}(\mathbf{u})^{\prime} \cdot \mathbf{u}^{\prime}}=0 .
$$


This situation happens, for instance, when we consider the Coriolis force $\mathbf{f}(\mathbf{u})=$ $2 \Omega \times \mathbf{u}$. In this case, the same procedure used to derive (22) allows us to write, in view of (23),

$$
\frac{\partial k}{\partial t}+\overline{\mathbf{u}} \cdot \nabla k=\operatorname{div}\left(v_{D}(k) \nabla k\right)+v_{T}(k)|\mathbf{D}(\overline{\mathbf{u}})|^{2}-\epsilon(k) .
$$

\section{A Stationary Problem}

In this section, we consider a stationary version of the problem formulated by the Eqs. (8), (21), and (24),

$$
\begin{aligned}
\operatorname{div} \mathbf{u} & =0, \\
\operatorname{div}(\mathbf{u} \otimes \mathbf{u}) & =\mathbf{g}-\mathbf{f}(\mathbf{u})-\frac{1}{\rho} \nabla p+\operatorname{div}\left(\left(v+v_{T}(k)\right) \mathbf{D}(\mathbf{u})\right), \\
\mathbf{u} \cdot \nabla k & =\operatorname{div}\left(v_{D}(k, \epsilon) \nabla k\right)+v_{T}|\mathbf{D}(\mathbf{u})|^{2}+g-\epsilon(k),
\end{aligned}
$$

where for the sake of simplifying the notation, we have omitted the bars over the filtered quantities. Observe also that in the last but one term of Eq. (27), we are considering a more general situation than in (24). We shall consider the problem posed by the Eqs. (25)-(27) in a bounded domain $\Omega \subset \mathbb{R}^{d}, d=2$, 3, with a compact boundary denoted by $\partial \Omega$. The problem (25)-(27) is supplemented by the following Dirichlet boundary conditions:

$$
\mathbf{u}=\mathbf{0}, \quad k=0 \quad \text { on } \partial \Omega .
$$

For the analysis we make in this work, we assume the turbulent viscosity and the turbulent diffusivity are bounded

$$
0 \leq v_{T}(k) \leq C_{T}, \quad c_{D} \leq v_{D}(k) \leq C_{D},
$$

where $C_{T}, c_{D}$, and $C_{D}$ are positive constants. The following weak formulation of the problem gives us the notion of the solutions we are interested in to look for.

Definition 1. Let $\Omega$ be a bounded domain of $\mathbb{R}^{d}, d=2,3$, and assume that both conditions in (29) are fulfilled. In addition, assume that

$$
\mathbf{g} \in \mathbf{L}^{2}(\Omega) \quad \text { and } \quad g \in L^{q}(\Omega) \text { with } \frac{2 d}{d+2} \leq q<d^{\prime}
$$

We say the couple $(\mathbf{u}, k)$ is a weak solution to the problem (25)-(28), if $\mathbf{u} \in \mathbf{V}$, $k \in W_{0}^{1, q}(\Omega)$, with $\frac{2 d}{d+2} \leq q<d^{\prime}, \mathbf{f}(\mathbf{u}) \cdot \mathbf{v} \in \mathbf{L}^{1}(\Omega)$ and 


$$
\int_{\Omega}((\mathbf{u} \cdot \nabla) \mathbf{u}) \cdot \mathbf{v} d \mathbf{x}+\int_{\Omega}\left(v+v_{T}(k)\right) \mathbf{D}(\mathbf{u}): \nabla \mathbf{v} d \mathbf{x}+\int_{\Omega} \mathbf{f}(\mathbf{u}) \cdot \mathbf{v} d \mathbf{x}=\int_{\Omega} \mathbf{g} \cdot \mathbf{v} d \mathbf{x}
$$

for any $\mathbf{v} \in \mathbf{V} \cap \mathbf{L}^{d}(\Omega), \varepsilon(k) \varphi \in L^{1}(\Omega)$ and

$$
\begin{aligned}
& \int_{\Omega}(\mathbf{u} \cdot \nabla k) \varphi d \mathbf{x}+\int_{\Omega} v_{D}(k) \nabla k \cdot \nabla \varphi d \mathbf{x}+\int_{\Omega} \varepsilon(k) \varphi d \mathbf{x} \\
= & \int_{\Omega} v_{T}(k)|\mathbf{D}(\mathbf{u})|^{2} \varphi d \mathbf{x}+\int_{\Omega} g \varphi d \mathbf{x}
\end{aligned}
$$

for any $\varphi \in W_{0}^{1, q^{\prime}}(\Omega)$, with $q^{\prime}>d$, and $k \geq 0$ and $\varepsilon \geq 0$ a.e. in $\Omega$.

In $[6,7]$ we prove two distinct existence results for the problem (25)-(28) in the sense of Definition 1. For the first existence result, we assume growth conditions both on the feedback $\mathbf{f}(\mathbf{u})$ and on the function $\varepsilon(k)$ that describe the turbulent dissipation (see [6]). For the second, we consider the case in which these terms are strongly nonlinear, i.e., without assuming any restrictions on its growth (see [7]). We have already established local higher integrability results for the gradients of $\mathbf{u}$ and of $k$.

Both proofs use an iterative scheme to uncouple the Navier-Stokes equations from the equation for the turbulent kinetic energy. The analysis of the decoupled equations follows the approach of $[1,2]$, with respect to the truncation of the feedbacks, and the arguing of [3] for the treatment of the $L^{1}$ terms.

Theorem 1. Let $\Omega$ be a bounded domain of $\mathbb{R}^{d}, d=2$, 3, with a Lipschitzcontinuous boundary $\partial \Omega$, and let $(\mathbf{u}, k)$ be a weak solution to the problem (25)-(28) in the conditions of Definition 1.

1. If $\mathbf{g} \in \mathbf{L}^{r}(\Omega)$, with $r>2$, and $\mathbf{f}(\mathbf{u}) \leq C|\mathbf{u}|^{s}$ for $0 \leq s \leq \frac{d+2}{d-2}$ if $d \neq 2$ or any $s \geq 0$ if $s=2$, then there exists $\sigma>2$ such that $\nabla \mathbf{u} \in \mathbf{L}^{\sigma}(\Omega)$.

2. If $g \in L^{r}(\Omega)$, with $r>d^{\prime}$, and $|\varepsilon(k)| \leq C|k|^{s}$ with $0 \leq s \leq 2-\frac{2(d-2)}{d(d-1)}$ if $d \neq 2$ or any $s \geq 0$ if $d=2$, then there exists $\tau>\frac{d}{d-1}$ such that $\nabla k \in L^{\tau}(\Omega)$ as long as $\nabla \mathbf{u} \in \mathbf{L}^{\sigma}(\Omega)$ for $\sigma>\frac{2 d(d-1)}{(d-1)^{2}+1}$.

The following result of global higher integrability that, for the reason of lack of space, cannot be shown here will be proved elsewhere.

The proof of this result adapts the arguments of [16] (see also [8]) for the NavierStokes equations together with the reasoning of [13] (see also [3]) for the equation for the turbulent kinetic energy. 


\section{On the Uniqueness}

We will prove the uniqueness by imposing conditions on the monotony of $\mathbf{f}(\mathbf{u})$ and $\varepsilon(k)$, as well as by imposing the Lipschitz continuity of $v_{T}(k)$ e $v_{D}(k)$.

Theorem 2. Let $\Omega$ be a bounded domain of $\mathbb{R}^{d}, d=2$, 3, with a Lipschitzcontinuous boundary $\partial \Omega$, and let $(\mathbf{u}, k)$ be a weak solution to the problem (25)-(28) in the conditions of Theorem 1. If the following conditions are fulfilled for all $\mathbf{u}_{1}, \mathbf{u}_{2} \in \mathbf{V}$ and for all $k_{1}, k_{2} \in W_{0}^{1, q}(\Omega)$,

$$
\begin{aligned}
& \left(\mathbf{f}\left(\mathbf{u}_{1}\right)-\mathbf{f}\left(\mathbf{u}_{2}\right)\right) \cdot\left(\mathbf{u}_{1}-\mathbf{u}_{2}\right) \geq 0, \quad\left(\varepsilon\left(k_{1}\right)-\varepsilon\left(k_{2}\right)\right)\left(k_{1}-k_{2}\right) \geq 0, \\
& \left|v_{T}\left(k_{1}\right)-v_{T}\left(k_{2}\right)\right| \leq C_{L_{1}}\left|k_{1}-k_{2}\right|, \quad\left|v_{D}\left(k_{1}\right)-v_{D}\left(k_{2}\right)\right| \leq C_{L_{2}}\left|k_{1}-k_{2}\right|,
\end{aligned}
$$

where $C_{L_{1}}$ and $C_{L_{2}}$ are positive constants and then the weak solution $(\mathbf{u}, k)$ is unique.

Remark 1. As we shall see in the proof, the above result is obtained under smallness assumptions on $\|\nabla \mathbf{u}\|_{\mathbf{L}^{\sigma}(\Omega)}$ and $\|\nabla k\|_{L^{\tau}(\Omega)}$ for $\sigma, \tau>2$ if $d=2$ or $\sigma, \tau \geq 2$ if $d \neq$ 2 , when compared with the kinematic viscosity $v$ and with the turbulent diffusivity lower bound $c_{D}$ [see $(29)_{2}$ ].

Proof. Being $\left(\mathbf{u}_{1}, k_{1}\right)$ and $\left(\mathbf{u}_{2}, k_{2}\right)$ two solutions of the problem, we start by subtracting the corresponding equation (31) of the weak formulation where in both is taken $\mathbf{v}:=\mathbf{u}_{1}-\mathbf{u}_{2}$ for the test function.

After some algebraic manipulations and using the assumptions (29) 2 and (33) together with Korn's inequality, we obtain

$$
\begin{aligned}
\frac{v}{C_{K}^{2}} \int_{\Omega}\left|\nabla\left(\mathbf{u}_{1}-\mathbf{u}_{2}\right)\right|^{2} d \mathbf{x} \leq & -\int_{\Omega}\left(v_{T}\left(k_{1}\right)-v_{T}\left(k_{2}\right)\right) \mathbf{D}\left(\mathbf{u}_{2}\right): \nabla\left(\mathbf{u}_{1}-\mathbf{u}_{2}\right) d \mathbf{x} \\
& -\int_{\Omega}\left(\left(\mathbf{u}_{1}-\mathbf{u}_{2}\right) \cdot \nabla\right) \mathbf{u}_{2} \cdot\left(\mathbf{u}_{1}-\mathbf{u}_{2}\right) d \mathbf{x}:=I_{1}+I_{2},
\end{aligned}
$$

where $C_{K}$ is the Korn's inequality constant. To estimate the term $I_{1}$, we use Hölder's and Sobolev's inequalities together with assertion 1 of Theorem 1 , which states that $\sigma>2$,

$$
\begin{aligned}
I_{1} & \leq\left\|k_{1}-k_{2}\right\|_{L^{2^{*}}(\Omega)}\left\|\nabla \mathbf{u}_{2}\right\|_{\mathbf{L}^{\sigma}(\Omega)}\left\|\nabla\left(\mathbf{u}_{1}-\mathbf{u}_{2}\right)\right\|_{\mathbf{L}^{2}(\Omega)} \\
& \leq C_{1}\left\|\nabla\left(k_{1}-k_{2}\right)\right\|_{L^{2}(\Omega)}\left\|\nabla\left(\mathbf{u}_{1}-\mathbf{u}_{2}\right)\right\|_{\mathbf{L}^{2}(\Omega)}, \quad C_{1}=C\left(d, \Omega,\left\|\nabla \mathbf{u}_{2}\right\|_{\mathbf{L}^{\sigma}(\Omega)}\right) .
\end{aligned}
$$

For $I_{2}$, we use Hölder's and Sobolev's inequalities, this in the case of $d \leq 4$, to obtain

$$
I_{2} \leq\left\|\mathbf{u}_{1}-\mathbf{u}_{2}\right\|_{\mathbf{L}^{2^{*}}(\Omega)}^{2}\left\|\nabla \mathbf{u}_{2}\right\|_{\mathbf{L}^{\sigma}(\Omega)} \leq C_{2}\left\|\nabla\left(\mathbf{u}_{1}-\mathbf{u}_{2}\right)\right\|_{\mathbf{L}^{2}(\Omega)}^{2},
$$


where $C_{2}=C\left(d, \Omega,\left\|\nabla \mathbf{u}_{2}\right\|_{\mathbf{L}^{\sigma}(\Omega)}\right)$. Now, gathering the estimates of $I_{1}$ and $I_{2}$ in (35), we obtain, after the use of Cauchy's inequality with suitable $\epsilon$,

$$
C_{\mathbf{u}}\left\|\nabla\left(\mathbf{u}_{1}-\mathbf{u}_{2}\right)\right\|_{\mathbf{L}^{2}(\Omega)}^{2} \leq C_{I}\left\|\nabla\left(k_{1}-k_{2}\right)\right\|_{L^{2}(\Omega)}^{2}, C_{\mathbf{u}}=\left(\frac{v}{2 C_{K}^{2}}-C_{2}\right), C_{I}=\frac{C_{1}^{2}}{2 v} .
$$

Next, we subtract the Eq. (32) corresponding to $k_{1}$ and $k_{2}$ and taking for test function, in both, $\varphi=k_{1}-k_{2}$. After some simplifications and using the assumptions $(29)_{1}$ and $(33)_{2}$, we obtain

$$
\begin{aligned}
& c_{D} \int_{\Omega}\left|\nabla\left(k_{1}-k_{2}\right)\right|^{2} d \mathbf{x} \leq-\int_{\Omega}\left(\mathbf{u}_{1} \cdot \nabla k_{1}-\mathbf{u}_{2} \cdot \nabla k_{2}\right)\left(k_{1}-k_{2}\right) d \mathbf{x} \\
& \quad-\int_{\Omega}\left(v_{D}\left(k_{1}\right)-v_{D}\left(k_{2}\right)\right) \nabla k_{2} \cdot \nabla\left(k_{1}-k_{2}\right) d \mathbf{x} \\
& \quad+\int_{\Omega}\left(v_{T}\left(k_{1}\right)\left|\mathbf{D}\left(\mathbf{u}_{1}\right)\right|^{2}-v_{T}\left(k_{2}\right)\left|\mathbf{D}\left(\mathbf{u}_{2}\right)\right|^{2}\right)\left(k_{1}-k_{2}\right) d \mathbf{x}:=J_{1}+J_{2}+J_{3} .
\end{aligned}
$$

After a simplification of $J_{1}$, we use Hölder's and Sobolev's inequalities, observing that $\tau>\frac{d}{d-1}$, to have

$$
\begin{aligned}
J_{1} \leq & \left\|\mathbf{u}_{1}\right\|_{\mathbf{L}^{\sigma}(\Omega)}\left\|\nabla\left(k_{1}-k_{2}\right)\right\|_{L^{2}(\Omega)}\left\|k_{1}-k_{2}\right\|_{L^{2^{*}}(\Omega)} \\
& +\left\|\mathbf{u}_{1}-\mathbf{u}_{2}\right\|_{\mathbf{L}^{2^{*}}(\Omega)}\left\|\nabla k_{1}\right\|_{L^{\tau}(\Omega)}\left\|\nabla\left(k_{1}-k_{2}\right)\right\|_{L^{2}(\Omega)} \\
\leq & C_{1}\left\|\nabla\left(k_{1}-k_{2}\right)\right\|_{L^{2}(\Omega)}^{2}+C_{2}\left\|\nabla\left(\mathbf{u}_{1}-\mathbf{u}_{2}\right)\right\|_{\mathbf{L}^{2}(\Omega)}\left\|\nabla\left(k_{1}-k_{2}\right)\right\|_{L^{2}(\Omega)} \\
\leq & C_{J_{1}}\left\|\nabla\left(k_{1}-k_{2}\right)\right\|_{L^{2}(\Omega)}^{2} \quad \text { by }(36), \quad C_{J_{1}}=C_{1}+C_{2} \sqrt{C_{I} / C_{\mathbf{u}}},
\end{aligned}
$$

where $C_{1}=C\left(d, \Omega,\left\|\nabla \mathbf{u}_{1}\right\|_{\mathbf{L}^{\sigma}(\Omega)}\right)$ and $C_{2}=C\left(d, \Omega,\left\|\nabla k_{1}\right\|_{L^{\tau}(\Omega)}\right)$. As for the term $J_{2}$, we use assumption $(34)_{2}$ together with Hölder's and Sobolev's inequalities, the last again in the case of $\tau>\frac{d}{d-1}$, in the following way

$$
J_{2} \leq C_{L_{2}}\left\|k_{1}-k_{2}\right\|_{L^{2^{*}}(\Omega)} \mid \nabla k_{2}\left\|_{\mathrm{L}^{\tau}(\Omega)}\right\| \nabla\left(k_{1}-k_{2}\right)\left\|_{\mathrm{L}^{2}(\Omega)} \leq C_{J_{2}}\right\| \nabla\left(k_{1}-k_{2}\right) \|_{L^{2}(\Omega)}^{2},
$$

where $C_{J_{2}}=C\left(C_{L_{2}}, d, \Omega,\left\|\nabla k_{2}\right\|_{\mathrm{L}^{\tau}(\Omega)}\right)$. The term $J_{3}$ is firstly simplified, and then we use the assumptions (29) and (34) 1 together with Hölder's and Sobolev's inequalities, and yet observing that $\sigma>2$,

$$
\begin{aligned}
J_{3} \leq & C_{L_{1}}\left\|k_{1}-k_{2}\right\|_{L^{2^{*}}(\Omega)}^{2}\left\|\nabla \mathbf{u}_{1}\right\|_{\mathbf{L}^{\sigma}(\Omega)}^{2} \\
& +C_{T}\left\|k_{1}-k_{2}\right\|_{L^{2^{*}}(\Omega)}\left\|\nabla\left(\mathbf{u}_{1}-\mathbf{u}_{2}\right)\right\|_{\mathbf{L}^{2}(\Omega)}\left\|\nabla \mathbf{u}_{1}+\nabla \mathbf{u}_{2}\right\|_{\mathbf{L}^{\sigma}(\Omega)} \\
\leq & C_{1}\left\|\nabla\left(k_{1}-k_{2}\right)\right\|_{L^{2}(\Omega)}^{2}+C_{2}\left\|\nabla\left(k_{1}-k_{2}\right)\right\|_{L^{2}(\Omega)}\left\|\nabla\left(\mathbf{u}_{1}-\mathbf{u}_{2}\right)\right\|_{\mathbf{L}^{2}(\Omega)} \\
\leq & C_{J_{3}}\left\|\nabla\left(k_{1}-k_{2}\right)\right\|_{L^{2}(\Omega)}^{2} \quad \text { by }(36), \quad C_{J_{3}}=C_{1}+C_{2} \sqrt{C_{I} / C_{\mathbf{u}}},
\end{aligned}
$$


where $C_{1}=C\left(C_{L_{1}}, d, \Omega,\left\|\nabla \mathbf{u}_{1}\right\|_{\mathbf{L}^{\sigma}(\Omega)}\right)$ and $C_{2}=C\left(C_{T}, \Omega,\left\|\nabla \mathbf{u}_{1}\right\|_{\mathbf{L}^{\sigma}(\Omega)},\left\|\nabla \mathbf{u}_{2}\right\|_{\mathbf{L}^{\sigma}(\Omega)}\right)$. Now, gathering the estimates of $J_{1}, J_{2}$, and $J_{3}$ in (37), we obtain $\left(c_{D}-C_{J}\right) \int_{\Omega} \mid \nabla\left(k_{1}-\right.$ $\left.k_{2}\right)\left.\right|^{2} d \mathbf{x} \leq 0$, where $C_{J}=\sum_{i=1}^{3} C_{J_{i}}$. As a consequence, it follows, by Sobolev's inequality, that $k_{1}=k_{2}$ a.e. in $\Omega$, as long as $c_{d}>C_{J}$. Consequently it follows from (36) that also $\mathbf{u}_{1}=\mathbf{u}_{2}$ a.e. in $\Omega$, as long as $v>2 C_{2} C_{K}^{2}$, where $C_{2}$ is the constant from the estimate of $I_{2}$.

\section{References}

1. Antontsev, S., Díaz, J.I., de Oliveira, H.B.: Stopping a viscous fluid by a feedback dissipative field: I. The stationary Stokes problem. J. Math. Fluid Mech. 6, 439-461 (2004)

2. Antontsev, S., Díaz, J.I., de Oliveira, H.B.: Stopping a viscous fluid by a feedback dissipative field: II. The stationary Navier-Stokes problem. Rend. Mat. Acc. Lincei s. 9 15, 257-270 (2004)

3. Boccardo, L., Gallouët, T.: Strongly nonlinear elliptic equations having natural growth terms and $L^{1}$ data. Nonlinear Anal. Theory Methods Appl. 19, 572-579 (1992)

4. Chacón-Rebollo, T., Lewandowski, R.: Mathematical and Numerical Foundations of Turbulence Models and Applications. Springer, New York (2014).

5. de Lemos, M. S.: Turbulence in Porous Media - Modelling and Applications. Elsevier, London (2012)

6. de Oliveira, H.B., Paiva, A.: Well-posedness of a stationary turbulent $k$-epsilon model with applications in porous media. Submitted.

7. de Oliveira, H.B., Paiva, A.: Existence for a one-equation turbulence $k$-epsilon model with strong nonlinearities. Submitted.

8. Druet, P.-E., Naumann, J., Wolf, J.: A Meyer's type estimate for weak solutions to a generalized stationary Navier-Stokes system. Humboldt-Universität, Berlin. Preprint 2008-6 (2008)

9. Kenjeres, S., Hanjalic, K.: On the implementation of effects of Lorentz force in turbulence closure models. Int. J. Heat Fluid 21, 329-337 (2000)

10. Kolmogorov, A.N.: In: Tikhomirov, V. (ed.) Selected Works of A. N. Kolmogorov, vol. 1. Kluwer Academic, Dordrecht (1991)

11. Launder, B., Spalding, D.: Mathematical Models of Turbulence. Academic, London (1972)

12. Mohammadi, B., Pironneau, O.: Analysis of the K-Epsilon Turbulence Model. Wiley-Masson, Paris (1993)

13. Naumann, J.: Existence of weak solutions to the equations of stationary motion of heatconducting incompressible viscous fluids. Progr. Nonlinear Differ. Equ. Appl. 64, 373-390 (2005)

14. Prandtl, L.: In: Oertel, H. (ed.) Prandtl-Essentials of Fluid Mechanics. Springer, New York (2008)

15. Rota, G.-C.: Reynolds Operators. In: Bellman, R. (ed.) Stochastic Processes in Mathematical Physics and Engineering, pp. 70-83. American Mathematical Society, Rhode Island (1964)

16. Wolff, M.: A global $L^{k}$-gradient estimate on weak solutions to nonlinear stationary NavierStokes equations under mixed boundary conditions. Humboldt-Universität, Berlin. Preprint 96-3 (1996) 